\title{
Fermi Arcs and Their Topological Character in the Candidate Type-II Weyl Semimetal MoTe 2
}

\author{
A. Tamai, ${ }^{1}$ Q. S. Wu, ${ }^{2}$ I. Cucchi, ${ }^{1}$ F. Y. Bruno, ${ }^{1}$ S. Riccò, ${ }^{1}$ T. K. Kim ${ }^{3}$ M. Hoesch, ${ }^{3}$ C. Barreteau, ${ }^{1}$ \\ E. Giannini, ${ }^{1}$ C. Besnard, ${ }^{4}$ A. A. Soluyanov, ${ }^{2,5}$ and F. Baumberger ${ }^{1,6}$ \\ ${ }^{1}$ Department of Quantum Matter Physics, University of Geneva, \\ 24 Quai Ernest-Ansermet, 1211 Geneva 4, Switzerland \\ ${ }^{2}$ Theoretical Physics and Station Q Zurich, ETH Zurich, 8093 Zurich, Switzerland \\ ${ }^{3}$ Diamond Light Source, Harwell Campus, Didcot OX11 ODE, United Kingdom \\ ${ }^{4}$ Laboratoire de cristallographie, Ecole de Physique, University of Geneva, \\ 24 Quai Ernest-Ansermet, 1211 Geneva 4, Switzerland \\ ${ }^{5}$ Department of Physics, St. Petersburg State University, St. Petersburg, 199034 Russia \\ ${ }^{6}$ Swiss Light Source, Paul Scherrer Institut, CH-5232 Villigen PSI, Switzerland
}

(Received 27 April 2016; revised manuscript received 14 June 2016; published 17 August 2016)

We report a combined experimental and theoretical study of the candidate type-II Weyl semimetal $\mathrm{MoTe}_{2}$. Using laser-based angle-resolved photoemission, we resolve multiple distinct Fermi arcs on the inequivalent top and bottom (001) surfaces. All surface states observed experimentally are reproduced by an electronic structure calculation for the experimental crystal structure that predicts a topological Weyl semimetal state with eight type-II Weyl points. We further use systematic electronic structure calculations simulating different Weyl point arrangements to discuss the robustness of the identified Weyl semimetal state and the topological character of Fermi arcs in $\mathrm{MoTe}_{2}$.

DOI: 10.1103/PhysRevX.6.031021

\section{INTRODUCTION}

The semimetallic transition metal dichalcogenides $(\mathrm{W} / \mathrm{Mo}) \mathrm{Te}_{2}$ receive much attention for their unusual bulk electronic properties, including a nonsaturating magnetoresistance with values among the highest ever reported [1] and pressure-induced superconductivity [2-4]. Very recent theoretical studies further predicted a new topological state of matter, dubbed type-II Weyl semimetal, in this series of compounds [5-8]. Weyl fermions in condensed matter arise as low-energy excitations at topologically protected crossing points (Weyl points) between electron and hole bands $[9,10]$. Weyl points always occur in pairs of opposite chirality, and their existence near the Fermi level produces unique physical properties, including different magnetotransport anomalies [5,11-15] and open Fermi arcs on the surface [9], which were not observed in early angleresolved photoemission (ARPES) studies [16-18].

Weyl semimetals have been classified in type I that exhibit pointlike Fermi surfaces and respect Lorentz invariance and type II with strongly tilted Weyl cones appearing at the boundaries between electron and hole Fermi pockets and low-energy excitations breaking Lorentz invariance [5]. Type-II Weyl semimetals necessarily have

Published by the American Physical Society under the terms of the Creative Commons Attribution 3.0 License. Further distribution of this work must maintain attribution to the author $(s)$ and the published article's title, journal citation, and DOI.
Subject Areas: Condensed Matter Physics, Materials Science, Topological Insulators extended bulk Fermi surfaces. This renders the identification of the topological character of the surface Fermi arcs challenging since the Weyl points generally lie within the surface projected bulk states causing strong hybridization of bulk and surface states where the latter overlap with the bulk continuum.

Experimentally, the realization of type-I Weyl fermions has been demonstrated in the TaAs family by ARPES and scanning tunneling spectroscopy [19-24], whereas the quest for type-II Weyl fermions remains open. Type-II Weyl fermions were first predicted in $\mathrm{WTe}_{2}$ [5] crystallizing in the orthorhombic $1 \mathrm{~T}^{\prime}$ structure with broken inversion symmetry (Pmn $2_{1}$ space group). According to this study, $\mathrm{WTe}_{2}$ hosts eight Weyl points $\sim 50 \mathrm{meV}$ above the Fermi level. However, the distance between Weyl points of opposite chirality is only $0.7 \%$ of the reciprocal lattice vector, rendering the observation of Fermi arcs connecting these points challenging for current spectroscopic techniques. Subsequently, it has been proposed that the lowtemperature $1 \mathrm{~T}^{\prime}$ phase of $\mathrm{MoTe}_{2}$ is a more robust type-II Weyl semimetal with topological Fermi arcs that are more extended in $k$ space $[6,7]$.

These predictions triggered a number of very recent ARPES studies reporting Fermi arcs on $\mathrm{Mo}_{x} \mathrm{We}_{1-x} \mathrm{Te}_{2}$ [25], $\mathrm{MoTe}_{2}$ [26-30], and $\mathrm{WTe}_{2}$ [31-33]. However, these studies do not agree on the bulk or surface character of the experimental Fermi surfaces, and provide conflicting interpretations of the topological character of the surface states. The latter can in part be attributed to the theoretically 
predicted sensitivity of the number and arrangement of Weyl points in the Brillouin zone to the details of the crystal structure. Two such experimentally measured structures were discussed for $\mathrm{MoTe}_{2}$ [6,7]. While the crystal structure used in the work of Refs. [6,30] was predicted to have eight type-II Weyl points, appearing in the $k_{z}=0$ plane [6], accompanied by 16 off-plane Weyl points [7], the structure reported in the work of Ref. [7] was predicted to host only four type-II Weyl points formed by the valence and conduction bands. The differences in the Weyl point arrangements lead to distinct topological characters of Fermi arcs with similar dispersion. These crystal structure intricacies, as well as the presence of two inequivalent surfaces in the noninversion symmetric structure of $\mathrm{MoTe}_{2}$, were largely ignored in recent works [25-30].

Here, we use laser-based ARPES to clearly resolve distinct arclike surface states on the two inequivalent (001) surfaces of $\mathrm{MoTe}_{2}$. To understand the topological nature of these arcs and their connection to type-II Weyl points, we present systematic calculations of the surface density of states simulating different Weyl point arrangements. We find that the ARPES data from both surfaces are consistent with a topological Weyl semimetal state with eight type-II Weyl points but do not conclusively establish this particular number of Weyl points. Specifically, we show that a large Fermi arc present on both surfaces and reported previously in Refs. [26-30] is topologically trivial in our calculations and even persists for bulk band structures without Weyl points. On one of the surfaces we find additional small Fermi arcs extending out of the hole pockets. These short arcs are strong candidates for the topological surface states. However, they resemble observations of Ref. [30] for a different Weyl point arrangement and we argue that they are not robust in the sense that their emergence out of the bulk continuum is not topologically protected.

\section{METHODS}

Single crystals of $\mathrm{MoTe}_{2}$ in the monoclinic $\beta$ phase were grown by an optimized chemical vapor transport method (See the Appendix A). The low-temperature $1 \mathrm{~T}^{\prime}$ crystal structure was characterized using single-crystal x-ray diffraction (Rigaku Supernova diffractometer, Mo $\mathrm{K} \alpha$ radiation, Oxford Instrument cryojet cooling system). Synchrotron-based ARPES experiments were performed at the I05 beamline of Diamond Light Source using photon energies of 40-90 eV. Laser-ARPES experiments were performed with a frequency-converted diode laser (LEOS solutions) providing continuous-wave radiation with 206-nm wavelength $(h \nu=6.01 \mathrm{eV})$ focused in a spot of $\sim 5-\mu \mathrm{m}$ diameter on the sample surface and an electron spectrometer (MB scientific) permitting two-dimensional $k$-space scans without rotating the sample. Samples were cleaved in situ along the $a b$ plane at temperatures $<20 \mathrm{~K}$. Measurement temperatures ranged from 6 to $20 \mathrm{~K}$ and the energy and momentum resolutions were $\sim 15 \mathrm{meV} /$ $0.02 \AA^{-1}$ and $2 \mathrm{meV} / 0.003 \AA^{-1}$ for synchrotron and laser-ARPES experiments, respectively. Electronic structure calculations were done using the VASP software package [34] with projector augmented wave pseudopotentials [35] that included spin-orbit coupling. The Perdew-Burke-Ernzerhof functional [36] was used in the exchange-correlation potential. A $16 \times 10 \times 4 \Gamma$-centered $k$-point mesh was used for Brillouin zone sampling, and the energy cutoff was set to $450 \mathrm{eV}$. The Wannier-based projected tight-binding models [37-39] capturing all the $d$ states of Mo and $p$ states of Te were used to analyze the surface density of states. Surface spectra were calculated by the software package Wannier_tools [40], which is based on the iterative Green function [41].

\section{RESULTS}

The monoclinic $\beta$ phase of $\mathrm{MoTe}_{2}$ undergoes a structural phase transition to the orthorhombic $1 \mathrm{~T}^{\prime}$ phase (also called $\gamma$ or $\mathrm{T}_{d}$ phase) at $\sim 250 \mathrm{~K}$ [42]. The low-temperature $1 \mathrm{~T}^{\prime}$ phase shares the noncentrosymmetric $P m n 2_{1}$ space group with $\mathrm{WTe}_{2}$ and consists of double layers of buckled tellurium atoms bound together by interleaving molybdenum atoms. The resulting $\mathrm{MoTe}_{2}$ layers are stacked along the $c$ axis with van der Waals interlayer bonding as illustrated in Fig. 1(a). Note that the broken inversion symmetry of the $1 \mathrm{~T}^{\prime}$ structure implies the existence of two inequivalent (001) surfaces with the [100] axis pointing out of or into the surface, respectively. We denote these surfaces by $A$ and $B$, as indicated in Fig. 1(a). In Figs. 1(b) and 1(c), we illustrate the number and positions
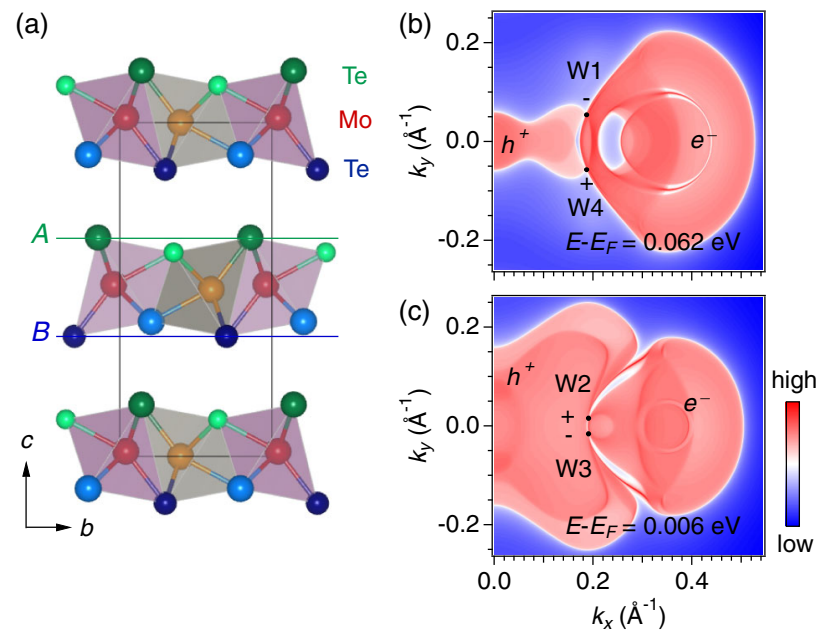

FIG. 1. Crystal structure and Weyl points of $\mathrm{MoTe}_{2}$. (a) Crystal structure of the $1 \mathrm{~T}^{\prime}$ phase (space group $P m n 2_{1}$ ) determined by $\mathrm{x}$-ray diffraction. The two inequivalent surfaces, labeled $A$ and $B$, are indicated. (b),(c) Momentum-resolved density of states of the (001) surface at $E-E_{F}=0.062$ and $0.006 \mathrm{eV}$, respectively, illustrating the touching points $\mathrm{W} 1,4$ and $\mathrm{W} 2,3$ of electron and hole pockets. W1(W2) is a mirror image of W4(W3). 
of Weyl points in $\mathrm{MoTe}_{2}$, calculated for the experimental crystal structure of our samples with lattice constants $a=3.468 \AA, b=6.310 \AA, c=13.861 \AA$ determined at $\mathrm{T}=100 \mathrm{~K}$ (see Figs. 6 and 7 for the temperature dependence of the lattice constants and calculations with unit cell parameters extrapolated to different temperatures).

Unlike Ref. [7] that predicted four Weyl points for a structure with $0.3 \%$ smaller lattice constant $a$, our new calculation finds eight type-II Weyl points between the valence and conduction band in the $k_{z}=0$ plane. We checked that no further off-plane Weyl points are present in our calculation. Note, however, that additional Weyl points above the Fermi level formed solely by the conduction bands were found in Ref. [7] (some of them in the $k_{z}=0$ plane). Given that the gaps separating different bands are small in $\mathrm{MoTe}_{2}$, even weak external perturbations may cause changes in band ordering, resulting in the appearance of additional Weyl points. This illustrates the exceptional sensitivity of key topological properties of $\mathrm{MoTe}_{2}$ to minute changes in the structure, as already pointed out in Refs. [6,7]. This problem is aggravated by the variation of the lattice constants of $\mathrm{MoTe}_{2}$ grown under different conditions, which might arise from a slight off stoichiometry and/or the proximity of the hexagonal $2 \mathrm{H}$ phase in the nonlinear temperature-composition phase diagram of $\mathrm{MoTe}_{2}$ favoring intergrowth of different phases [43].

In Fig. 2, we present the overall band structure of $\mathrm{MoTe}_{2}$ determined by ARPES. The Fermi surface consists of a large holelike sheet centered at the $\Gamma$ point and two symmetric electronlike Fermi surfaces, one at positive and one at negative $k_{x}$ values, that nearly touch the hole pocket. The character of the different Fermi surfaces can be inferred from the stack of constant energy maps in Fig. 2(a) illustrating how the sizes of electron and hole pockets decrease and increase with energy, respectively. The dominant spectral feature at the Fermi surface is a symmetric arclike contour in the narrow gap between electron and hole pockets [black line in Fig. 2(b)], which is not seen in bulk band structure calculations. The $k_{x}-k_{z}$ Fermi surface map in Fig. 2(c) shows that this state (SS) does not disperse in $k_{z}$ over the entire Brillouin zone as expected for a strictly two-dimensional electronic state localized at the surface. Figure 2(d) shows the overall band dispersion along the high-symmetry direction $k_{y}=0$. The surface state dispersing from the bottom of the electron bands up to the hole bands is indicated by a thin red line. The same cut measured with high-resolution laser ARPES (blue inset) reveals a more complex situation. Rather than a single surface state, we can resolve two very sharp dispersing states with similar Fermi velocities. While the outer one at larger $k_{x}$ values shows a clear Fermi level crossing, the inner one, which is most intense $\sim 100 \mathrm{meV}$ below $E_{F}$, loses most of its spectral weight approaching $E_{F}$.

In Fig. 3, we zoom into the region enclosed by a red dashed rectangle in Fig. 2(b). Using a laser focused into a spot of $\sim 5-\mu \mathrm{m}$ diameter on the sample surface as excitation
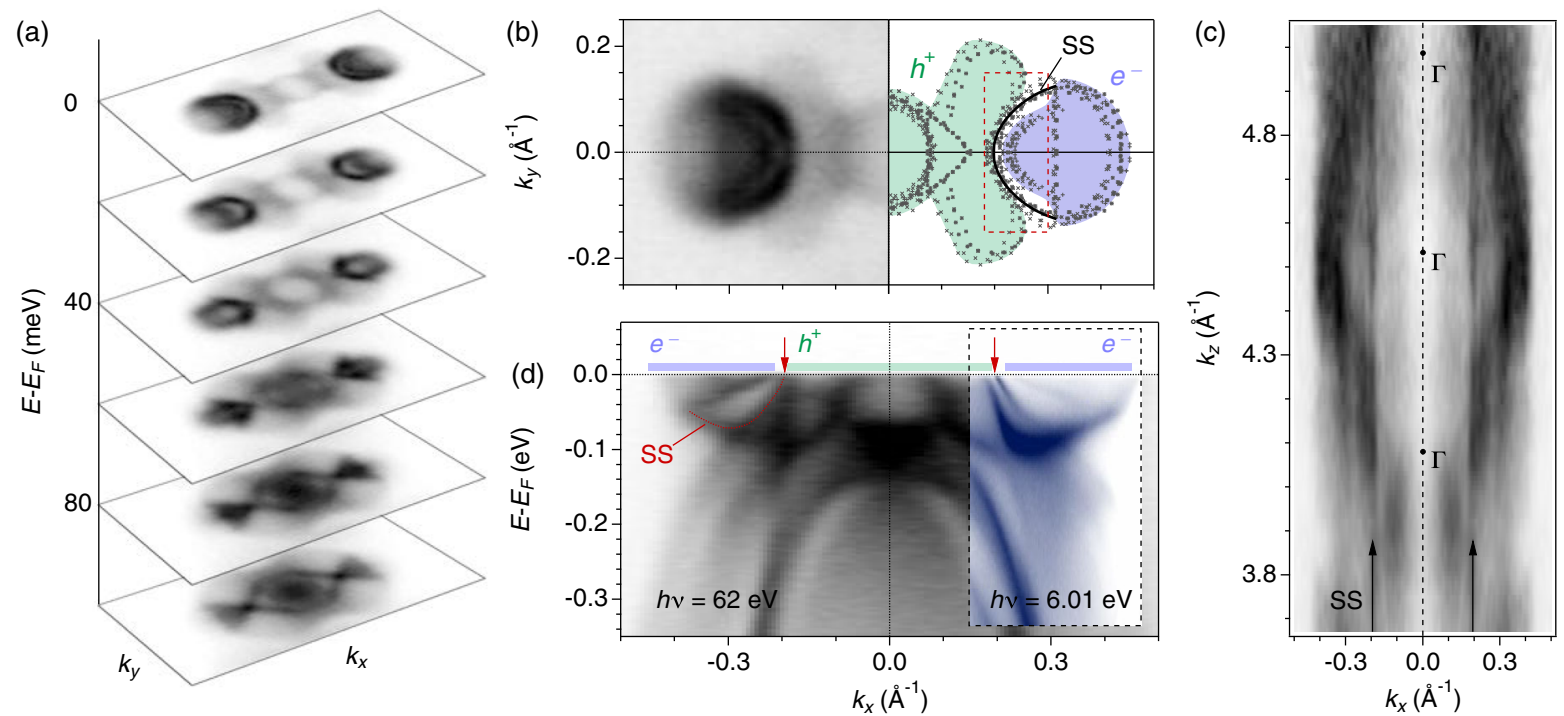

FIG. 2. Electronic structure of $\mathrm{MoTe}_{2}$. (a) Stack of constant energy maps measured with $62 \mathrm{eV}$ photon energy and $p$ polarization. (b) Left: ARPES Fermi surface at $62 \mathrm{eV}$ photon energy. Right: Fermi surface contours extracted from Fermi surface maps measured at different photon energies. The largest extension of electron and hole pockets corresponding to the projected bulk band structure is indicated in blue and green, respectively. The thin black arc between hole and electron pockets is a surface state. (c) $k_{z}$ dependence of the electronic states at the Fermi level in the $k_{y}=0$ plane. The straight lines at $k_{x}= \pm 0.195 \AA^{-1}$ marked by black arrows are the signature of a two-dimensional electronic state at the surface of $\mathrm{MoTe}_{2}$. Values of $k_{z}$ are calculated for free-electron final states with an inner potential of $13 \mathrm{eV}$. (d) Overview of the surface band structure measured along the high-symmetry direction $k_{y}=0$ taken with $h \nu=62$ and $6.01 \mathrm{eV}$ (blue inset). 

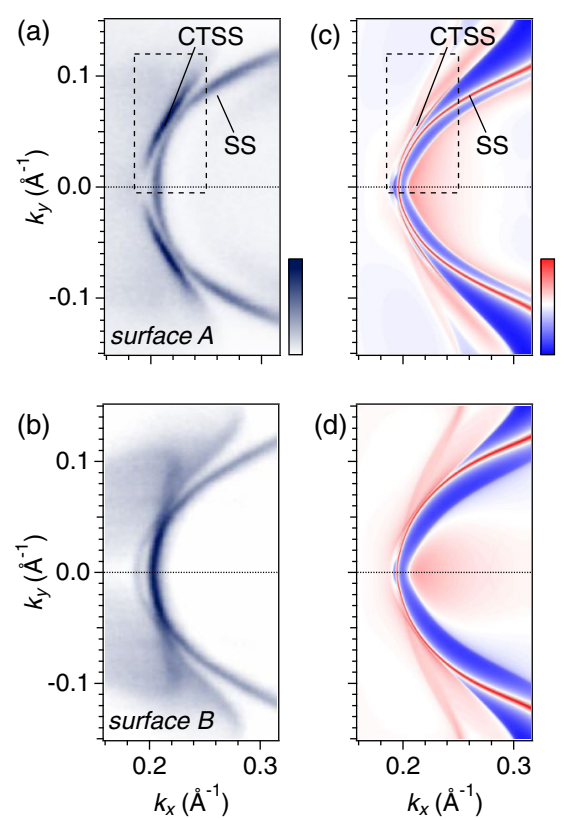
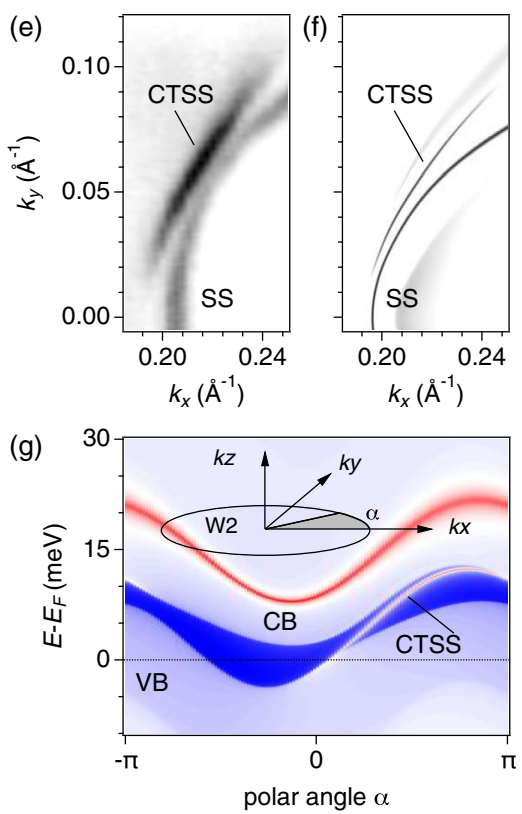

FIG. 3. Fermi arcs on the surface of $\mathrm{MoTe}_{2}$. (a),(b) Detailed view of the Fermi surface measured with $6.01 \mathrm{eV}$ photon energy and $p$ polarization on the $A$ and $B$ surface terminations of $\mathrm{MoTe}_{2}$. The photoemission intensity has been symmetrized with respect to the $k_{x}$ axis. (c),(d) Surface density of states for the two surface terminations calculated $10 \mathrm{meV}$ below the Fermi level. (e),(f) Zoom into the areas enclosed by a dashed rectangle in (a) and (c), respectively. (g) Numerical results for the surface density of states along an elliptical contour (with $r_{k x}=0.0018 \AA^{-1}, r_{k y}=0.005 \AA^{-1}$ ) enclosing the Weyl point W2. The topological surface state is connecting valence to conduction bands and produces CTSS in the calculations.

source and high energy and momentum resolution, we are able to identify two clearly distinct Fermi surfaces appearing with the same abundance on a large number of cleaved samples. For surface $A$ we find two sets of intense and sharp contours, a large arclike state (SS) and two shorter arcs with weak curvature that are visible in a narrow range of momenta $\left|k_{y}\right| \lesssim 0.08 \AA^{-1}$ and vanish again in the $k_{y}=0$ plane [Fig. 3(a)]. On the $B$-type surface, we resolve two large arcs with different curvatures separated by a small noncrossing gap [Fig. 3(b)]. These signatures are well reproduced by calculations of the surface density of states for the inequivalent top and bottom surface of $\mathrm{MoTe}_{2}$ shown in Figs. 3(c) and 3(d).

According to the calculations presented in Fig. 3 and in the Appendix C, the large Fermi arc (SS) is topologically trivial for the present crystal structure, and even persists in calculations for different structures that exhibit no bulk Weyl points at all. The shorter arc (CTSS for candidate topological surface state), on the other hand, is a part of the surface state that connects valence to conduction states, as shown for the elliptical path surrounding the projection of the W2 point onto the surface [Fig. 3(g)]. This elliptical path can be viewed as a cut of a cylinder that encloses the W2 point, and the axis of which is aligned along [001]. The valence and conduction bands are gapped at all $k$ points on this cylinder, and thus a Chern number can be computed for the occupied states, corresponding to the chirality of the enclosed Weyl point. We find this number to be +1 , and hence CTSS in Fig. 3(g) is topologically protected. The calculation agrees remarkably well with the experiment [Figs. 3(e) and 3(f)]. Therefore, CTSS is a strong candidate for a topological surface state.

The good agreement between ARPES data and numerical calculations extends to the dispersion plots shown in Figs. 4(a) and 4(b) taken parallel to the $k_{y}$ axis for different values of $k_{x}$. The trivial surface state SS has a nearly parabolic dispersion in our experiments and well-defined Fermi crossings for $k_{x} \geq 0.21 \AA^{-1}$, whereas CTSS has a more complex evolution of the dispersion and reaches the Fermi level only over a very limited range of momenta [second and third panels of Fig. 4(a)]. This behavior is well reproduced by the calculated surface density of states [Fig. 4(b)], showing that CTSS is defined only for energies and momenta where it emerges out of the projected bulk hole pocket. For this reason it is not possible to derive the positions of type-II Weyl points from the extensions of the topological arcs at the Fermi surface.

We note that the intensity of the bulk bands is strongly suppressed in our laser-ARPES data. This is common in very low-energy photoemission and arises from the bandlike character of final states leading to strongly photonenergy-dependent matrix elements of bulk states. We further find that all bulk states are broad compared to SS and CTSS. This can be explained naturally by the surface sensitivity of photoemission causing an intrinsically poor $k_{z}$ resolution. For the large unit cell of $\mathrm{MoTe}_{2}$, we 

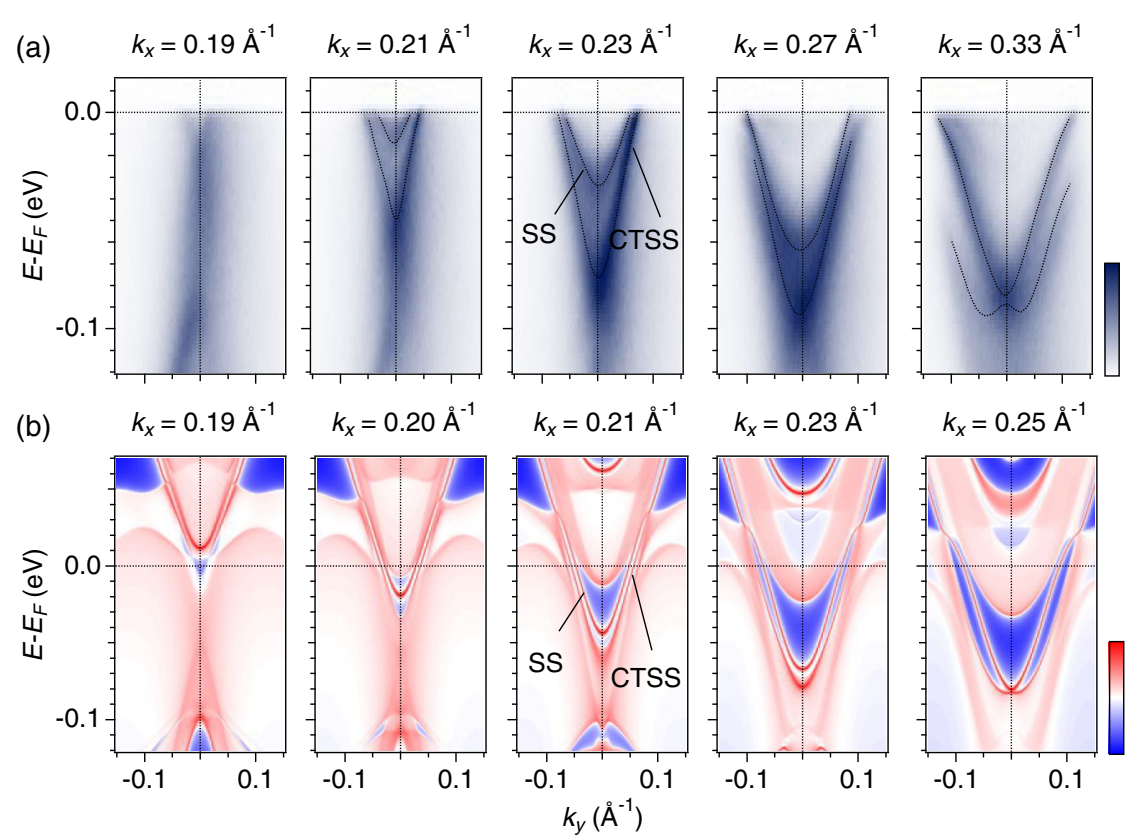

FIG. 4. (a) Dispersion plots taken on surface $A$ parallel to $k_{y}$ for different values of $k_{x}$. (b) Calculated surface energy dispersion along equivalent cuts in momentum space for the same surface termination.

estimate that $\Delta k_{z}>0.2 \pi / c$, which implies a substantial broadening of states that disperse in $k_{z}$. The absence of such broadening in CTSS indicates that this state is highly two dimensional, consistent with its identification as a surface state.

CTSS found in the calculations connects Weyl points that both project into the bulk hole pocket at the Fermi level. Moreover, all the Fermi pockets in $\mathrm{MoTe}_{2}$ have zero Chern numbers. For these reasons the emergence of Fermi arcs from the hole pocket is not dictated by topology, but is rather a consequence of a particular realization of the shape of CTSS. In general, a topologically trivial surface state can also emerge out of the topologically trivial pocket, as is the case at hand for SS. The intricate relation of CTSS to the Weyl points of the system is evident from the fact that a Fermi arc with very similar dispersion was also reported in the calculations of Refs. [6,30] with different in-plane Weyl point positions and 16 additional off-plane Weyl points [7]. In that case, additional surface resonances or surface states hybridized with each other can appear inside or outside the nearby bulk continuum due to the presence of the off-plane Weyl points. In principle, such resonances may cause the observed state to be topologically trivial in analogy to SS. On the other hand, CTSS in our calculations and its analogue in Refs. [6,30] are both topological, and we do not find surface states with similar shape that are topologically trivial in any of our calculations. The question is therefore whether the absence of a topologically trivial scenario at hand can be taken as positive proof of a Weyl semimetal state in $\mathrm{MoTe}_{2}$. The systematic nature of calculations carried out by us and others suggests that this is reasonable even though evidence of the absence (in this case of a topologically trivial scenario explaining CTSS) is never exhaustive.

CTSS is observed clearly on only one of the two inequivalent (001) surfaces. This can be explained by noticing that the trivial value of the $\mathbb{Z}_{2}$ topological invariant [44] for the $k_{y}=0$ plane in the Brillouin zone of $\mathrm{MoTe}_{2}$ is consistent with two possible arc connectivities: the one consistent with CTSS, assuming that a surface state connects the projections of $\mathrm{W} 1$ and $\mathrm{W} 2$ points, and the other, in which the arcs connect the projections of W1,2 with their mirror images W3,4. While the ARPES data of the $B$-type surface [Fig. 3(b)] are consistent with the latter scenario, they do not uniquely define a particular Weyl point arrangement and connectivity.

To illustrate this point, we show in Figs. 5(c) and 5(d) the surface density of states on the $B$-type surface for crystal structures resulting in four [7] and eight (this work) type-II Weyl points. Both calculations clearly show a large and fully spin-polarized Fermi arc with virtually identical dispersion. However, in the structure of Ref. [7] with four Weyl points [Fig. 5(c)], this arc is topological, while for the crystal structure of this work, we find that the arc is trivial [Fig. 5(d)]. As shown in Figs. 10 and 11, the large Fermi arc persists even in calculations for $\mathrm{MoTe}_{2}$ and $\mathrm{WTe}_{2}$ finding zero Weyl points. This demonstrates that the topological character of Fermi arcs in $(\mathrm{Mo} / \mathrm{W}) \mathrm{Te}_{2}$ cannot, in general, be uniquely deduced from a comparison of experimental and theoretical band dispersions. It further shows that neither the experimental observation of the large Fermi arc in $\mathrm{MoTe}_{2}$ or $\mathrm{WTe}_{2}$ nor of its spin structure is a suitable fingerprint to robustly identify the type-II Weyl state in this series of compounds. The observation of CTSS, on the 

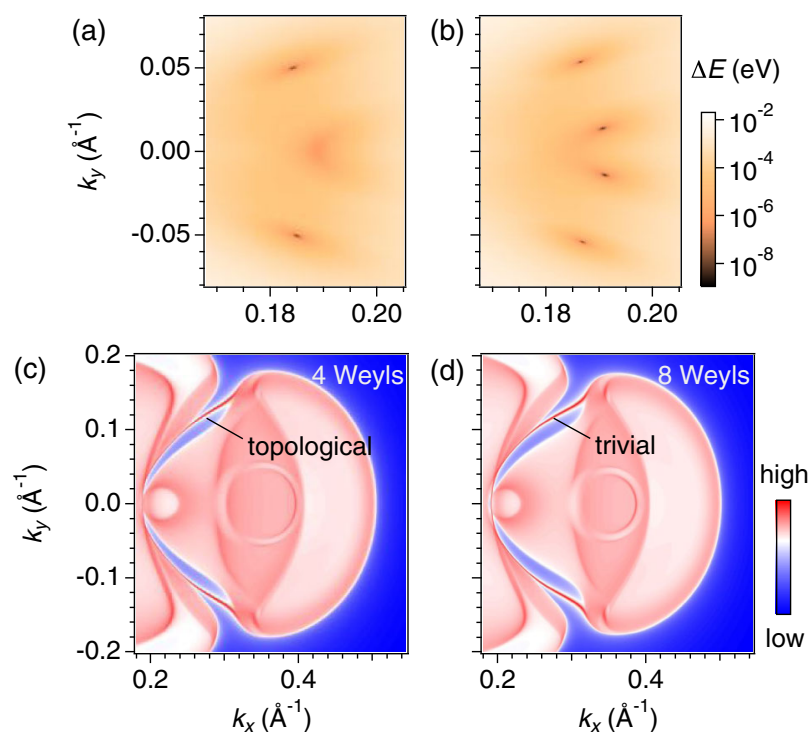

FIG. 5. Trivial and topological Fermi arcs on the $B$-type surface of $\mathrm{MoTe}_{2}$. (a) Position of the four Weyl points calculated for the experimental crystal structure reported in Ref. [7]. (b) Using the structure determined in our work, we find eight Weyl points. (c),(d) Theoretical Fermi surfaces for the structures used in (a) and (b), respectively. The large Fermi arc has a virtually identical dispersion in the two scenarios but is topological in (c) and trivial in $(\mathrm{d})$.

other hand, cannot readily be explained in a topologically trivial scenario and thus does provide evidence for a type-II Weyl semimetal state.

\section{CONCLUSIONS}

We conclude that $\mathrm{MoTe}_{2}$ is a strong candidate for the realization of a type-II Weyl semimetal phase. All the experimental observations presented here agree with our numerical calculations finding eight type-II Weyl points and a coexistence of topological and trivial Fermi arcs. While the data are also consistent with other possible Weyl point arrangements, systematic calculations do not show a topologically trivial scenario that could explain all the experimental observations. We point out that an ideal candidate type-II Weyl semimetal permitting a more direct experimental identification should possess projected electron and hole pockets with nonzero total Chern number, thus guaranteeing the appearance of the surface state in both numerical and experimental data.

\section{ACKNOWLEDGMENTS}

We gratefully acknowledge discussions with G. Autés, A. Bernevig, R. Cerny, A. Morpurgo, and C. Renner. This work was supported by the Swiss National Science Foundation (200021-146995, 200021-153405, PZ00P2161327). Q. S. W. and A. A. S. acknowledge funding from Microsoft Research and the Swiss National Science
Foundation through the National Competence Centers in Research MARVEL and QSIT. Q. S. W. was also supported by the National Natural Science Foundation of China (11404024). The authors acknowledge Diamond Light Source for time on beamline I05 under proposal SI12404.

\section{APPENDIX A: CRYSTAL STRUCTURE AND NUMBER OF WEYL POINTS}

\section{Crystal growth}

Single crystals of monoclinic $\beta-\mathrm{MoTe}_{2}$ are grown by the chemical vapor transport technique using $\mathrm{TeCl}_{4}$ as a transport agent. The pure elements $\mathrm{Mo}$ and $\mathrm{Te}$ and the transport agent $\mathrm{TeCl}_{4}$ are mixed in a stoichiometric anion ratio, according to the reaction equation

$2\left[\left(\frac{n-1}{n}\right) \mathrm{Te}+\left(\frac{1}{n}\right) \mathrm{TeCl}_{4}\right]+\mathrm{Mo} \rightarrow \mathrm{MoTe}_{2}+\left(\frac{4}{n}\right) \mathrm{Cl}_{2}$,

with $n=10$. The total weight of each sample is $\sim 0.2-0.3 \mathrm{~g}$. The mixture is weighted in a glovebox and sealed under vacuum $\left(5 \times 10^{-6}\right.$ mbar $)$ in a quartz ampule with an internal diameter of $8 \mathrm{~mm}$ and a length of $120 \mathrm{~mm}$. The sealed quartz reactor is placed in a two-zone furnace in the presence of a thermal gradient $d T / d x \approx 5^{\circ}-10^{\circ} \mathrm{C} / \mathrm{cm}$, and heated up to temperatures $T_{\text {hot }}=980^{\circ} \mathrm{C}$ at the hot end and $T_{\text {cold }}=900^{\circ} \mathrm{C}$ at the cold end. After one week of growth, the quartz ampule is quenched in air to yield the monoclinic phase, $\beta-\mathrm{MoTe}_{2}$. The crystals obtained on the cold side are shiny gray and rectangular.

\section{Lattice constants and Weyl points}

The monoclinic $\beta$ phase of $\mathrm{MoTe}_{2}$ (unit cell parameters at $280 \mathrm{~K}$ of $a=3.479 \AA, b=6.332 \AA, c=13.832 \AA$, $\gamma=93.83^{\circ}$ ) undergoes a structural phase transition to the orthorhombic $1 \mathrm{~T}^{\prime}$ phase at $\sim 250 \mathrm{~K}$. Figure 6 displays the

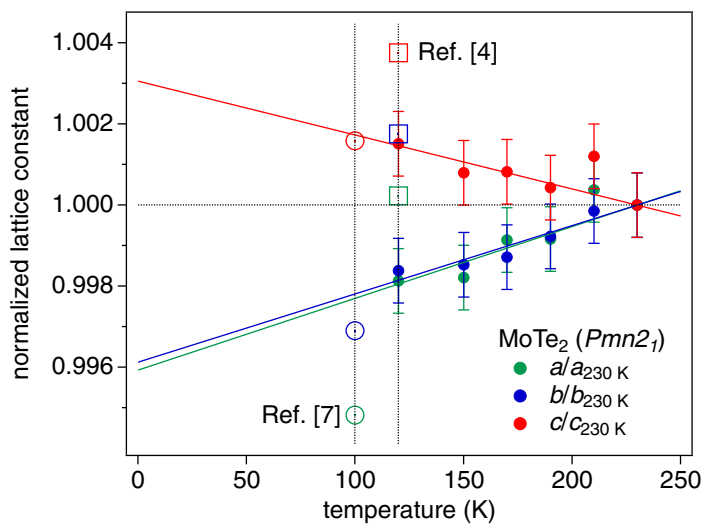

FIG. 6. Temperature dependence of the lattice constants of $\mathrm{MoTe}_{2}$ in the $1 \mathrm{~T}^{\prime}$ phase (space group $P m n 2_{1}$ ). Open circles and squares are the lattice constants of $\mathrm{MoTe}_{2}$ obtained with different sample growth procedures as reported in Refs. [4,7]. All values have been normalized by the lattice parameters of our samples measured at $230 \mathrm{~K}(a=3.4762 \AA, b=6.3239 \AA, c=13.837 \AA)$. 


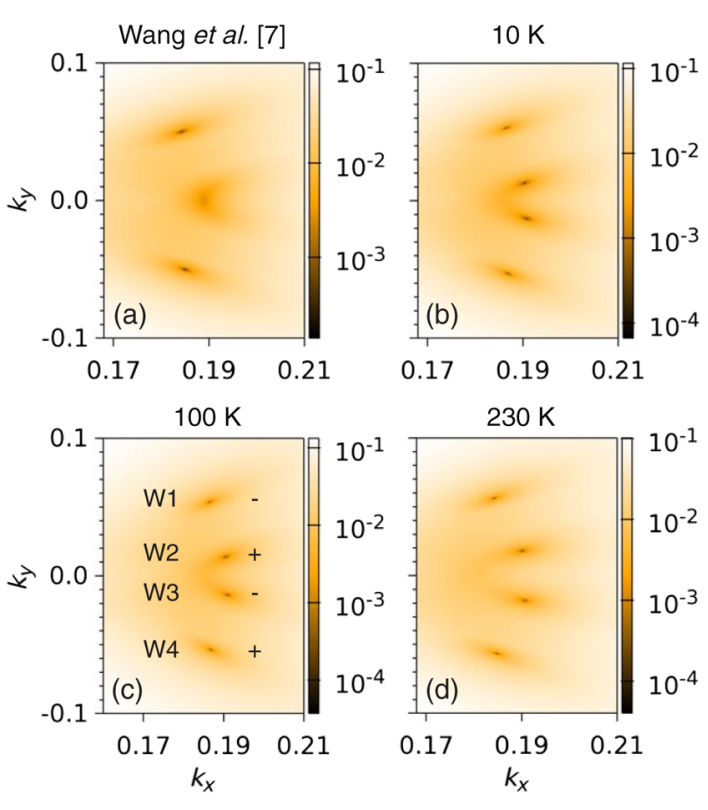

FIG. 7. False-color plot of the energy gap between valence and conduction bands in the $k_{z}=0$ plane illustrating how the number and position of Weyl points evolve for different unit cell parameters: (a) Structure in Ref. [7] which has a $0.3 \%$ smaller lattice constant $a$; (b)-(d) lattice constants at 10, 100, and $230 \mathrm{~K}$ obtained from a linear extrapolation based on the experimental data in Fig. 11. The coordinates of the Weyl points found for the unit cell parameters at $100 \mathrm{~K}$ used in the main text are $(0.1855, \pm 0.0539,0) \AA^{-1}$ and $E-E_{F}=0.062 \mathrm{eV}$ for $\mathrm{W} 1,4$ and $(0.1906, \pm 0.0152,0) \AA^{-1}$ and $E-E_{F}=0.006 \mathrm{eV}$ for $\mathrm{W} 2,3$. The remaining four points are mirror symmetric with respect to $k_{x}$

temperature dependence of the lattice constants in the $1 \mathrm{~T}^{\prime}$ phase of $\mathrm{MoTe}_{2}$. Consistent with the early study of Clarke et al. [42], we find that the $c$ axis expands with decreasing temperature while $a$ and $b$ decrease slightly. For comparison, we also display the lattice constants of $\mathrm{MoTe}_{2}$ from the two previously reported structures in Refs. [4,7].

In the main text, we compare ARPES data measured below $20 \mathrm{~K}$ with calculations based on the lattice constants at $100 \mathrm{~K}$ extrapolated linearly from the experimental values measured between 230 and $120 \mathrm{~K}$. This is a reasonable approach as we do not expect significant changes of the unit cell parameters between $100 \mathrm{~K}$ and the temperature of the ARPES experiments. The thermal expansion coefficient is usually negligible below $\sim 100 \mathrm{~K}$, and no additional structural phase transitions have been reported for $\mathrm{MoTe}_{2}$ at these low temperatures. In order to confirm the validity of this comparison, we performed additional calculations for different sets of lattice parameters assuming constant thermal expansion. As shown in Figs. 7(b)-7(d), calculations based on the lattice constants of our structure at 10 , 100 , and $230 \mathrm{~K}$ estimated in this way find the same number of Weyl points at nearly identical positions. All these structures result in eight Weyl points at $k_{z}=0$ and no additional off-plane Weyl points, formed between valence and conduction bands. This indicates that the contraction of the lattice upon cooling will have only a minor effect in our samples and will not change the topological character of the Fermi arcs discussed in the main text. Note, however, that Fig. 7 reveals a clear trend in the position and number of Weyl points with the in-plane lattice constant $a$. As $a$ gets smaller, due to thermal contraction [Figs. 7(b)-7(d)] or a different growth method [Fig. 7(a)], the Weyl points W2 and W3 move closer to the $k_{x}$ axis and will eventually merge and annihilate.

\section{APPENDIX B: SURFACE STATE DISPERSION PARALLEL TO THE $\boldsymbol{k}_{\boldsymbol{y}}=0$ DIRECTION}

In Fig. 8, we present dispersion plots measured parallel to the $k_{y}=0$ direction on the two different surfaces of $\mathrm{MoTe}_{2}$. In each panel of Figs. 8(d) and 8(f) we can identify two dispersing states that contribute to the trivial arc (SS) and the candidate topological arc (CTSS) at the Fermi surface. Their dispersion is clearly distinct on the two surface types. In particular, on the $A$-type surface, SS is more dispersive near the bottom of the bulk electron pocket and the two surface states cross each other along the highsymmetry cut with $k_{y}=0$, while this is not observed on the $B$-type surface. These differences are well reproduced by calculations of the surface band structure for a semi-infinite crystal [Figs. 8(c) and 8(e)], allowing us to unambiguously identify surface $A$ and $B$ as the top and bottom (001) surface of $\mathrm{MoTe}_{2}$.

Figure 8 also provides further evidence for the surface character of CTSS. In particular, the clearly distinct dispersion of this spectral feature below the Fermi level is hard to reconcile with a bulk origin, since bulk states are not expected to differ between the two surface types. Additionally, we note that all bulk states are substantially broader and much less intense than surface-related states throughout the entire Brillouin zone. This is evident from Figs. 8(a) and 8(b) where we use a logarithmic color scale to enhance the projected bulk Fermi surfaces.

\section{APPENDIX C: TOPOLOGICAL CHARACTER OF FERMI ARCS}

\section{Candidate topological Fermi arc}

In Fig. 3(g), we show that the candidate topological surface state (CTSS) identified in our ARPES data connects the valence (hole) and conduction (electron) band along a fully gapped path enclosing a single Weyl point with Chern number +1 (W2) and is thus topologically nontrivial. In Fig. 9, we use more conventional $k$-space maps at different, finely spaced energies to illustrate how this state connects the valence and conduction bands. At the Fermi level, a small gap separates the momentum-resolved surface density of states of the electron and hole pockets and the CTSS arc emerges on both ends out of the hole pocket. As the 


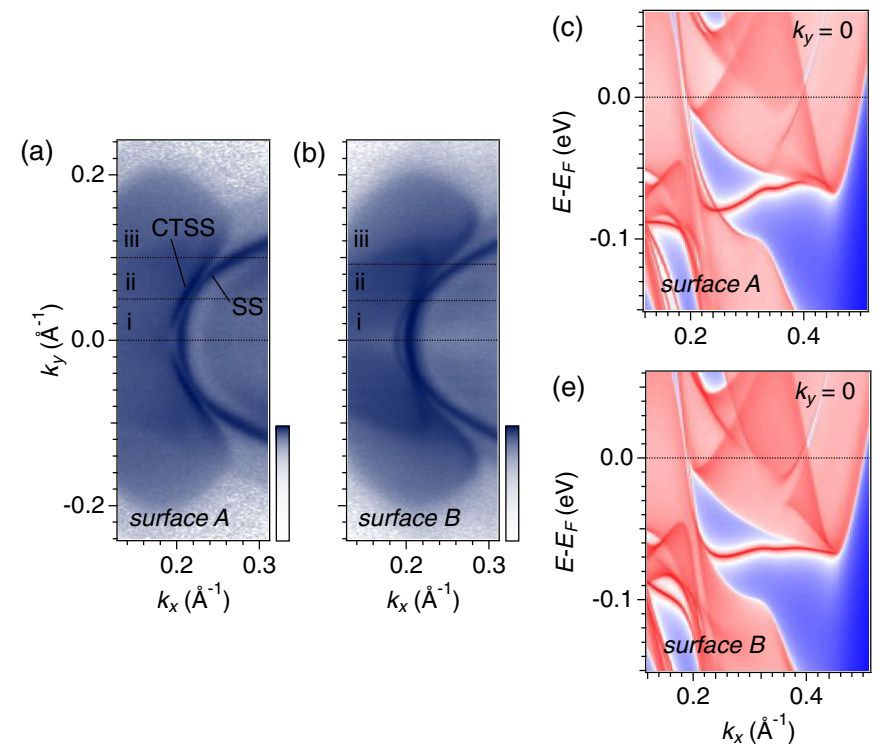

(d) $\operatorname{surface} A$
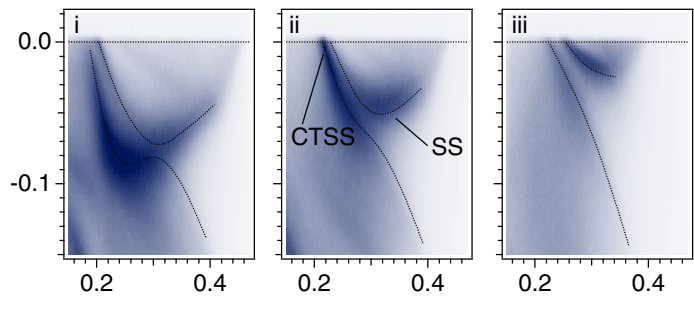

(f) surface $B$
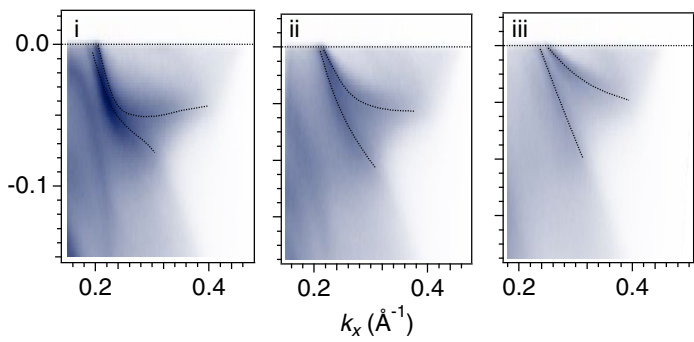

FIG. 8. (a),(b) Detailed view of the Fermi surface measured with $6.01 \mathrm{eV}$ photon energy and $p$ polarization on the $A$ - and $B$-type surfaces of $\mathrm{MoTe}_{2}$, displayed on a logarithmic color scale. This photon energy corresponds to $k_{z}$ values around $0.6 \pi / c$ in the fifth Brillouin zone assuming a free-electron final state with an inner potential of $13 \mathrm{eV}$. Note, however, that a free-electron final state model is not fully appropriate at such low photon energies, which results in a substantial uncertainty of $k_{z}$ values. (c),(e) Surface energy dispersion along the $k_{y}=0$ direction calculated for the top and bottom surface, respectively. (d),(f) Dispersion plots measured on surface $A$ and $B$ parallel to the $k_{y}=0$ direction at the $k_{y}$ values "i"-"iii" marked in (a) and (b), respectively.

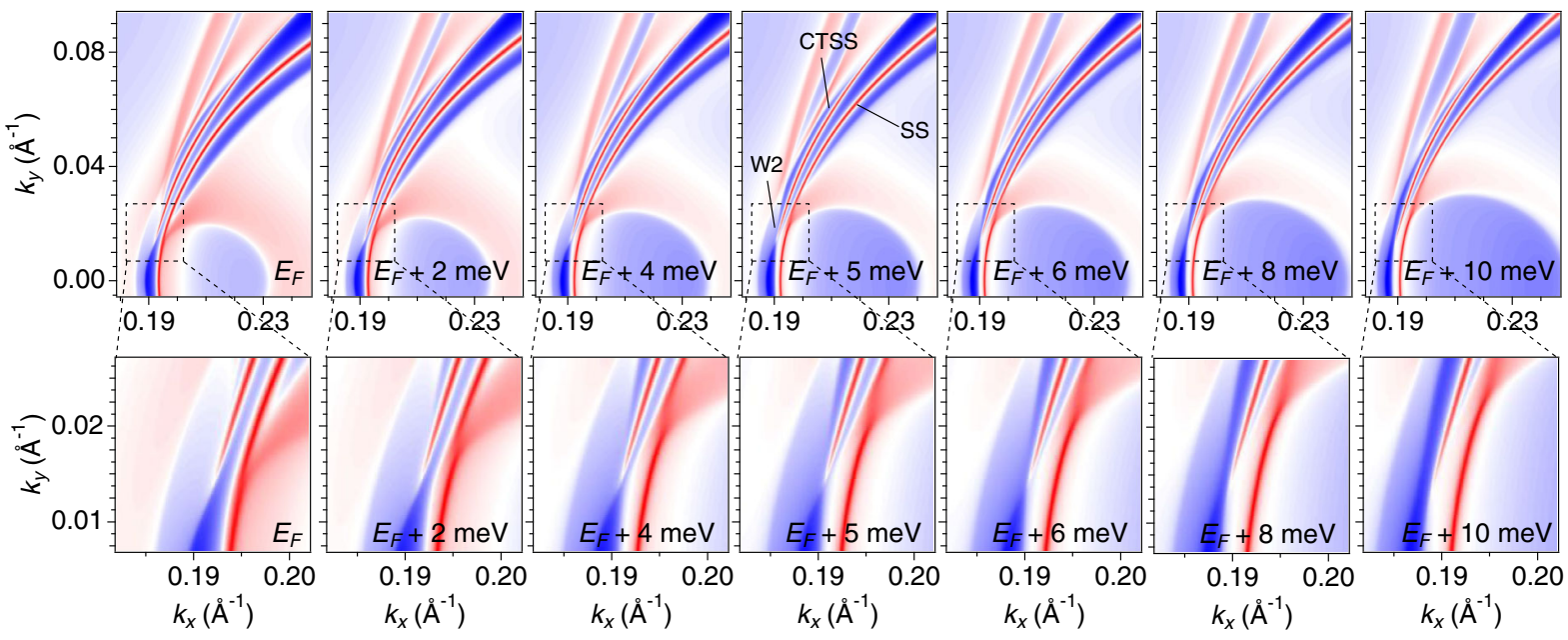

FIG. 9. Momentum-resolved surface density of states illustrating the evolution of the topological Fermi arc (CTSS) around the energy of the W2 Weyl point.

energy is increased towards the Weyl point $\mathrm{W} 2$ at $+6 \mathrm{meV}$, this gap reduces and the electron and hole pockets eventually touch. At higher energy, the CTSS arc emerges out of the electron pocket at $k_{y} \approx 0.015 \AA^{-1}$ and disappears into the hole pocket at $k_{y} \approx 0.07 \AA^{-1}$.

\section{Large Fermi arc}

We show in the main text that the number of Weyl points in the Brillouin zone and the topological character of the large Fermi arc in $\mathrm{MoTe}_{2}$ are exceptionally sensitive to small changes in the crystal structure as they are observed for samples grown under slightly different conditions. In Fig. 10, we use an alternative approach to illustrate the persistence of the large Fermi arc for different Weyl point arrangements in $\mathrm{MoTe}_{2}$. By artificially tuning the strength of the spin-orbit coupling (SOC) (the labels SOCN correspond to SOC increased $N$ times in the calculation), we simulate electronic structures with 8,4 , or 0 Weyl points for a single crystal structure. Clearly, the large Fermi arc is observed in all these calculations. Its presence in experiment [25-30] thus cannot serve as a fingerprint to identify $\mathrm{MoTe}_{2}$ as a Weyl semimetal. 

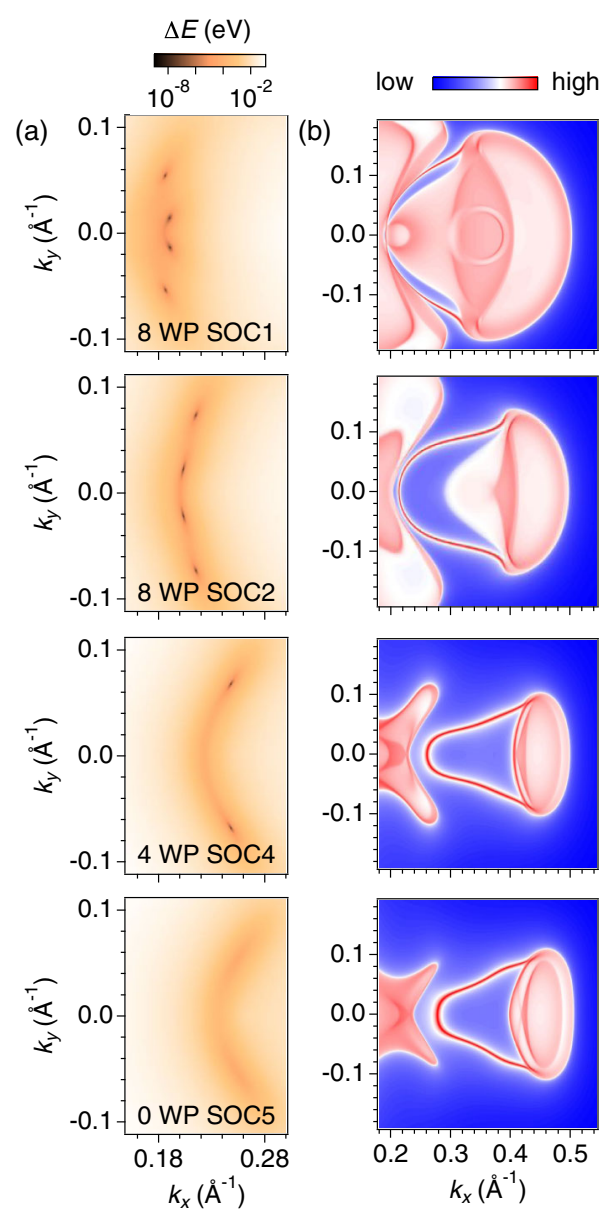

FIG. 10. Evolution of the large Fermi arcs for different values of the spin-orbit coupling (SOC) constant, which artificially modify the band structure of $\mathrm{MoTe}_{2}$ resulting in 8,4 , or 0 Weyl points (WP) in the $k_{z}=0$ plane. (a) False-color plot of the energy gap between valence and conduction bands in the $k_{z}=0$ plane illustrating the number and position of Weyl points. (b) Momentum-resolved surface density of states at the Fermi level.

The independence of the large Fermi arc from the Weyl point arrangement is even more striking in $\mathrm{WTe}_{2}$, as shown in Fig. 11. In Figs. 11(a) and 11(c), we show the momentumresolved surface density of states for experimental crystal structures measured at different temperatures [45,46]. Warming up from $113 \mathrm{~K}$ to room temperature, one can readily annihilate the eight Weyl points found for the lowtemperature structure [5]. However, the large Fermi arc remains present and does not even change its dispersion noticeably. Artificially increasing the spin-orbit interaction, we can even simulate a state with a large Fermi arc of similar dispersion and four Weyl points in the full 3D Brillouin zone [Fig. 11(b)]. In this case, the large Fermi arc becomes topological, while in the other two calculations [Figs. 11(a) and 11(c)], it is topologically trivial. Again, we conclude that its observation in experiments [31-33] does not identify $\mathrm{WTe}_{2}$ as a topological Weyl semimetal, as discussed already in Ref. [31].
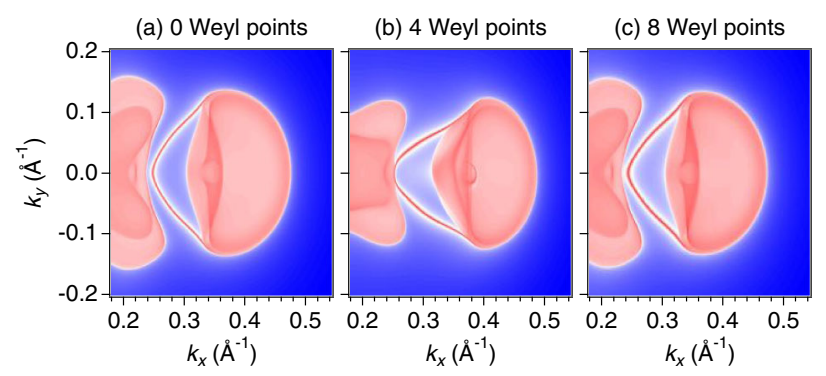

FIG. 11. Large Fermi arc of $\mathrm{WTe}_{2}$ for different numbers of Weyl points. (a) Momentum-resolved surface density of states at the Fermi level calculated using the room temperature structure [45], which results in no Weyl points. (b) Momentum-resolved surface density of states at the Fermi level calculated using the low-temperature structure ( $T=113 \mathrm{~K}$, Ref. [46]) and 200\% spin-orbit coupling, which produces only four Weyl points. (c) Momentum-resolved surface density of states at the Fermi level calculated using the low-temperature structure $(T=113 \mathrm{~K}$ Ref. [46]) and 100\% spin-orbit coupling, which produces eight Weyl points.

The large Fermi arcs in $\mathrm{MoTe}_{2}$ and $\mathrm{WTe}_{2}$ remain fully spin polarized in all our calculations although they are, in general, topologically trivial except for the calculations with four Weyl points. The lifting of the spin degeneracy is a natural consequence of spin-orbit interaction in structures with a broken inversion symmetry and is thus found for all surface and bulk states in the $1 \mathrm{~T}^{\prime}$ phase of $\mathrm{MoTe}_{2}$ and $\mathrm{WTe}_{2}$. Hence, the topological character of this and other surface states in $\mathrm{MoTe}_{2}$ or $\mathrm{WTe}_{2}$ cannot be deduced from their spin structure, as it was proposed in Ref. [30] and successfully done for topological insulators [47].

[1] M. N. Ali, J. Xiong, S. Flynn, J. Tao, Q. D. Gibson, L. M. Schoop, T. Liang, N. Haldolaarachchige, M. Hirschberger, N. P. Ong, and R. J. Cava, Large, Non-Saturating Magnetoresistance in $\mathrm{WTe}_{2}$, Nature (London) 514, 205 (2014).

[2] X.-C. Pan, X. Chen, H. Liu, Y. Feng, Z. Wei, Y. Zhou, Z. Chi, L. Pi, F. Yen, F. Song, X. Wan, Z. Yang, B. Wang, G. Wang, and Y. Zhang, Pressure-Driven Dome-Shaped Superconductivity and Electronic Structural Evolution in Tungsten Ditelluride., Nat. Commun. 6, 7805 (2015).

[3] D. Kang, Y. Zhou, W. Yi, C. Yang, J. Guo, Y. Shi, S. Zhang, Z. Wang, C. Zhang, S. Jiang, A. Li, K. Yang, Q. Wu, G. Zhang, L. Sun, and Z. Zhao, Superconductivity Emerging from a Suppressed Large Magnetoresistant State in Tungsten Ditelluride., Nat. Commun. 6, 7804 (2015).

[4] Y. Qi et al., Superconductivity in Weyl Semimetal Candidate $\mathrm{MoTe}_{2}$, Nat. Commun. 7, 11038 (2016).

[5] A. A. Soluyanov, D. Gresch, Z. Wang, Q. Wu, M. Troyer, X. Dai, and B. Andrei Bernevig, Type-II Weyl Semimetals, Nature (London) 527, 495 (2015).

[6] Y. Sun, S.-C. Wu, M. N. Ali, C. Felser, and B. Yan, Prediction of Weyl Semimetal in Orthorhombic $\mathrm{MoTe}_{2}$, Phys. Rev. B 92, 161107 (2015). 
[7] Z. Wang, D. Gresch, A. A. Soluyanov, W. Xie, S. Kushwaha, X. Dai, M. Troyer, R. J. Cava, and B. A. Bernevig, $\mathrm{MoTe}_{2}: A$ Type-II Weyl Topological semimetal, Phys. Rev. Lett. 117, 056805 (2016).

[8] T.-R. Chang, S.-Y. Xu, G. Chang, C.-C. Lee, S.-M. Huang, B. Wang, G. Bian, H. Zheng, D. S. Sanchez, I. Belopolski, N. Alidoust, M. Neupane, A. Bansil, H.-T. Jeng, H. Lin, and M. Zahid Hasan, Prediction of an Arc-Tunable Weyl Fermion Metallic State in $\mathrm{Mo}_{x} \mathrm{~W}_{1-x} \mathrm{Te}_{2}$, Nat. Commun. 7, 10639 (2016).

[9] X. Wan, A. M. Turner, A. Vishwanath, and S. Y. Savrasov, Topological Semimetal and Fermi-Arc Surface States in the Electronic Structure of Pyrochlore Iridates, Phys. Rev. B 83, 205101 (2011).

[10] H. Weng, C. Fang, Z. Fang, B. Andrei Bernevig, and X. Dai, Weyl Semimetal Phase in Noncentrosymmetric TransitionMetal Monophosphides, Phys. Rev. X 5, 011029 (2015).

[11] H. B. Nielsen and M. Ninomiya, The Adler-Bell-Jackiw Anomaly and Weyl Fermions in a Crystal, Phys. Lett. B 130, 389 (1983).

[12] D. T. Son and B.Z. Spivak, Chiral Anomaly and Classical Negative Magnetoresistance of Weyl Metals, Phys. Rev. B 88, 104412 (2013).

[13] P. Hosur and X. Qi, Recent Developments in Transport Phenomena in Weyl Semimetals, C.R. Phys. 14, 857 (2013).

[14] B. Z. Spivak and A. V. Andreev, Magnetotransport Phenomena Related to the Chiral Anomaly in Weyl Semimetals, Phys. Rev. B 93, 085107 (2016).

[15] A. A. Burkov, Chiral Anomaly and Transport in Weyl Metals, J. Phys. Condens. Matter 27, 113201 (2015).

[16] I. Pletikosić, M. N. Ali, A. V. Fedorov, R. J. Cava, and T. Valla, Electronic Structure Basis for the Extraordinary Magnetoresistance in $\mathrm{WTe}_{2}$, Phys. Rev. Lett. 113, 216601 (2014).

[17] J. Jiang, F. Tang, X. C. Pan, H. M. Liu, X. H. Niu, Y. X. Wang, D. F. Xu, H. F. Yang, B. P. Xie, F. Q. Song, P. Dudin, T. K. Kim, M. Hoesch, P. K. Das, I. Vobornik, X. G. Wan, and D. L. Feng, Signature of Strong Spin-Orbital Coupling in the Large Nonsaturating Magnetoresistance Material $\mathrm{WTe}_{2}$, Phys. Rev. Lett. 115, 166601 (2015).

[18] Y. Wu, N. H. Jo, M. Ochi, L. Huang, D. Mou, S. L. Bud'ko, P. C. Canfield, N. Trivedi, R. Arita, and A. Kaminski, Temperature-Induced Lifshitz Transition in $\mathrm{WTe}_{2}$, Phys. Rev. Lett. 115, 166602 (2015).

[19] S.-Y. Xu, I. Belopolski, N. Alidoust, M. Neupane, G. Bian, C. Zhang, R. Sankar, G. Chang, Z. Yuan, C.-C. Lee et al., Discovery of a Weyl Fermion Semimetal and Topological Fermi Arcs, Science 349, 613 (2015).

[20] B. Q. Lv, H. M. Weng, B. B. Fu, X. P. Wang, H. Miao, J. Ma, P. Richard, X. C. Huang, L. X. Zhao, G. F. Chen et al., Experimental Discovery of Weyl Semimetal TaAs, Phys. Rev. X 5, 031013 (2015).

[21] B. Q. Lv, N. Xu, H. M. Weng, J. Z. Ma, P. Richard, X. C. Huang, L. X. Zhao, G. F. Chen, C. Matt, F. Bisti, V. Strokov, J. Mesot, Z. Fang, X. Dai, T. Qian, M. Shi, and H. Ding, Observation of Weyl nodes in TaAs, Nat. Phys. 11, 724 (2015).

[22] L. X. Yang, Z. K. Liu, Y. Sun, H. Peng, H. F. Yang, T. Zhang, B. Zhou, Y. Zhang, Y.F. Guo, M. Rahn, D. Prabhakaran, Z. Hussain, S.-K. Mo, C. Felser, B. Yan, and Y.L. Chen, Weyl Semimetal Phase in the NonCentrosymmetric Compound TaAs, Nat. Phys. 11, 728 (2015).

[23] S.-Y. Xu et al., Discovery of a Weyl Fermion State with Fermi Arcs in Niobium Arsenide, Nat. Phys. 11, 748 (2015).

[24] H. Inoue, A. Gyenis, Z. Wang, J. Li, S. W. Oh, S. Jiang, N. Ni, B. Andrei Bernevig, and A. Yazdani, Quasiparticle Interference of the Fermi Arcs and Surface-Bulk Connectivity of a Weyl Semimetal, Science 351, 1184 (2016).

[25] Ilya Belopolski et al., Unoccupied Electronic Structure and Signatures of Topological Fermi Arcs in the Weyl Semimetal Candidate $\mathrm{Mo}_{x} \mathrm{~W}_{1-x} \mathrm{Te}_{2}$, arXiv:1512.09099.

[26] L. Huang, T. M. McCormick, M. Ochi, M.-T. Suzuki, R. Arita, Y. Wu, D. Mou, H. Cao, J. Yan, N. Trivedi, and A. Kaminski, Spectroscopic Evidence for Type II Weyl Semimetal State in $\mathrm{MoTe}_{2}$, arXiv:1603.06482v1.

[27] K. Deng, G. Wan, P. Deng, K. Zhang, S. Ding, E. Wang, M. Yan, H. Huang, H. Zhang, Z. Xu, J. Denlinger, A. Fedorov, H. Yang, W. Duan, H. Yao, Y. Wu, Y. Shoushan Fan, H. Zhang, X. Chen, and Shuyun Zhou, Experimental Observation of Topological Fermi Arcs in Type-II Weyl Semimetal $\mathrm{MoTe}_{2}$, arXiv:1603.08508.

[28] Aiji Liang et al., Electronic Evidence for Type II Weyl Semimetal State in $\mathrm{MoTe}_{2}$, arXiv:1604.01706.

[29] N. Xu, Z. J. Wang, A. P. Weber, A. Magrez, P. Bugnon, H. Berger, B. B. Fu, B. Q. Lv, N. C. Plumb, M. Radovic, K. Conder, T. Qian, J. H. Dil, J. Mesot, H. Ding, and M. Shi, Discovery of Weyl Semimetal State Violating Lorentz Invariance in $\mathrm{MoTe}_{2}$, arXiv:1604.02116.

[30] J. Jiang, Z. K. Liu, Y. Sun, H. F. Yang, R. Rajamathi, Y. P. Qi, L. X. Yang, C. Chen, H. Peng, C.-C. Hwang, S. Z. Sun, S.-K. Mo, I. Vobornik, J. Fujii, S. S. P. Parkin, C. Felser, B. H. Yan, and Y. L. Chen, Observation of the Type-II Weyl Semimetal Phase in $\mathrm{MoTe}_{2}$, arXiv:1604.00139.

[31] F. Y. Bruno, A. Tamai, Q. S. Wu, I. Cucchi, C. Barreteau, A. de la Torre, S. McKeown Walker, S. Riccò, Z. Wang, T. K. Kim, M. Hoesch, M. Shi, N. C. Plumb, E. Giannini, A. A. Soluyanov, and F. Baumberger, Surface States and Bulk Electronic Structure in the Candidate Type-II Weyl Semimetal $\mathrm{WTe}_{2}$, arXiv:1604.02411.

[32] C. Wang et al., Spectroscopic Evidence of Type II Weyl Semimetal State in $\mathrm{WTe}_{2}$, arXiv:1604.04218.

[33] Y. Wu, N. H. Jo, D. Mou, L. Huang, S. L. Bud'ko, P. C. Canfield, and A. Kaminski, Observation of Fermi Arcs in TypeII Weyl Semimetal Candidate $\mathrm{WTe}_{2}$, arXiv:1604.05176.

[34] G. Kresse and J. Furthmüller, Efficient Iterative Schemes for Ab Initio Total-Energy Calculations Using a Plane-Wave Basis Set, Phys. Rev. B 54, 11169 (1996).

[35] P. E. Blöchl, Projector Augmented-Wave Method, Phys. Rev. B 50, 17953 (1994).

[36] J.P. Perdew, K. Burke, and M. Ernzerhof, Generalized Gradient Approximation Made Simple, Phys. Rev. Lett. 77, 3865 (1996).

[37] N. Marzari and D. Vanderbilt, Maximally Localized Generalized Wannier Functions for Composite Energy Bands, Phys. Rev. B 56, 12847 (1997).

[38] I. Souza, N. Marzari, and D. Vanderbilt, Maximally Localized Wannier Functions for Entangled Energy Bands, Phys. Rev. B 65, 035109 (2001). 
[39] A. A. Mostofi, J. R. Yates, G. Pizzi, Y.-S. Lee, I. Souza, D. Vanderbilt, and N. Marzari, An Updated Version of WANNIER90: A Tool for Obtaining Maximally-Localised Wannier Functions, Comput. Phys. Commun. 185, 2309 (2014).

[40] Q.S. Wu, Wannier_Tools, https://github.com/quanshengwu/ wannier_tools.

[41] M. P. Lopez Sancho, J. M. Lopez Sancho, J. M. L. Sancho, and J. Rubio, Highly Convergent Schemes for the Calculation of Bulk and Surface Green Functions, J. Phys. F 15, 851 (1985).

[42] R. Clarke, E. Marseglia, and H. P. Hughes, A LowTemperature Structural Phase Transition in $\beta-\mathrm{MoTe}_{2}$, Philos. Mag. B 38, 121 (1978).

[43] L. Brewer and R. H. Lamoreaux, in Binary Alloys Phase Diagrams, edited by T. B. Massalski (ASM International, Ohio, 1990), Vol. 3, pp. 2675-2676.
[44] C. L. Kane and E. J. Mele, $Z_{2}$ Topological Order and the Quantum Spin Hall Effect, Phys. Rev. Lett. 95, 146802 (2005).

[45] B.E. Brown, The Crystal Structures of $\mathrm{WTe}_{2}$ and High-Temperature $\mathrm{MoTe}_{2}$, Acta Crystallogr. 20, 268 (1966).

[46] A. Mar, S. Jobic, and J. A. Ibers, Metal-Metal vs TelluriumTellurium Bonding in $\mathrm{WTe}_{2}$ and Its Ternary Variants TaIrTe $_{4}$ and $\mathrm{NbIrTe}_{4}$, J. Am. Chem. Soc. 114, 8963 (1992).

[47] D. Hsieh, Y. Xia, L. Wray, D. Qian, A. Pal, J. H. Dil, J. Osterwalder, F. Meier, G. Bihlmayer, C. L. Kane, Y. S. Hor, R. J. Cava, and M.Z. Hasan, Observation of Unconventional Quantum Spin Textures in Topological Insulators, Science 323, 919 (2009). 